\title{
Clarifying the importance of microplastic particles as vectors for long-range transport of chemical contaminants: a response to letter to the editor
}

\author{
Todd Gouin ${ }^{*}$
}

\begin{abstract}
This correspondence article provides a response to comments that have been raised regarding the long-range environmental transport of chemical additives associated with microplastic particles and plastic debris, which includes additional clarification for the continuing need for holistic approaches. Recognizing that not all microplastic particles are equal with respect to environmental fate and toxicological effects, it is strongly encouraged that the research community continue to acquire quantitative understanding regarding which types of microplastic particles are likely to be subject to long-range transport. Comments and responses that engage in constructive dialogue represent positive contributions that can only strengthen our ability to evaluate the role of microplastic and plastic debris as vectors of transport for plastic associated chemicals, and the associated risks, which represents an important component of the scientific process.
\end{abstract}

Keywords: Long-range environmental transport, Plastic additives, Exposure assessment

In my recent Perspective article [1], I explored the weight of evidence that microplastic particles (MPs) can act as vectors for the long range environmental transport (LRET) of plastic additive chemicals (PACs). The purpose of a Perspective article is to 'focus' attention on important issues and to provoke discussion; e.g. via the presentation of new proposals for tackling existing problems. I therefore welcome the comments from Glüge et al. [2] and the opportunity to engage in additional dialogue about the role of MPs as vectors of LRET for PACs.

Although nine shortcomings are identified [2], many of the points overlap and can be summarized under three headings:
1. The LRET of plastic debris $>5 \mathrm{~mm}$ is poorly addressed in the Perspective, which potentially fails to consider a significant component of the overall issue.

2. The leaching of PACs from plastic debris does not receive sufficient discussion and, therefore, the article fails to consider a significant component of the overall issue.

3. Concerns raised about the article published by Andrade et al. [3] are unfounded and unwarranted.

As a general observation, many of the specific concerns that are raised appear to reflect either a misunderstanding and/or a misinterpretation of the information presented by Gouin [1]. For instance, the Perspective [1] is entitled "Addressing the importance of microplastic particles as vectors for long-range transport of chemical contaminants". Thus, the intent of the Perspective was always to specifically focus on the role of MPs as vectors

${ }^{*}$ Correspondence: todd.gouin@environresearch.com

TG Environmental Research, 18 Wellpond Cl, Sharnbrook, Bedford MK44 $1 \mathrm{PL}, \mathrm{UK}$

(C) The Author(s) 2022. Open Access This article is licensed under a Creative Commons Attribution 4.0 International License, which permits use, sharing, adaptation, distribution and reproduction in any medium or format, as long as you give appropriate credit to the original author(s) and the source, provide a link to the Creative Commons licence, and indicate if changes were made. The images or other third party material in this article are included in the article's Creative Commons licence, unless indicated otherwise in a credit line to the material. If material is not included in the article's Creative Commons licence and your intended use is not permitted by statutory regulation or exceeds the permitted use, you will need to obtain permission directly from the copyright holder. To view a copy of this licence, visit http://creativecommons.org/licenses/by/4.0/. 
of LRET, not plastic debris $>5 \mathrm{~mm}$. This is further clarified at the end of the introduction [1]. Nevertheless, I was interested to learn that Glüge et al. [2] report that MPs may not be easily available for oceanic long-range transport, a statement that I think is perhaps critically important to communicate to the wider scientific community and the public. As Glüge et al. [2] are undoubtably aware, significant resources have been directed towards monitoring levels of MPs in surface ocean water, sediment, and in marine biota, which has resulted in considerable concern, particularly in the media, regarding the global impact of MPs. On the other hand, it may be that I am guilty of misinterpreting the statement made by Glüge et al. [2] regarding the LRET of MPs. It is conceivable that they may be arguing that macroplastic debris is more important than MPs in the context of LRET, since it is the macroplastic that is transported to remote locations where it may then be subject to degradation and fragmentation processes, resulting in the formation of MPs.

In fact, the Perspective [1] identifies degradation and fragmentation of larger plastic debris as a source of MPs to the environment that requires improved understanding. Thus, it seems beneficial to support the development of holistic mechanistic models seeking to integrate the interactions between a variety of complex processes. For instance, there is a need to include the transport of both macro- and microplastic AND the simultaneous processes of degradation, fragmentation, and sedimentation, all of which influence both categories of plastic debris. Additional processes, such as the formation of biofilms on the surface of plastic and the influence of heteroaggregation are also important processes to consider in the context of characterizing and quantifying environmental fate, mobility and exposure. This calls for better models of the environmental fate and transport of plastic debris and MPs, as governed by their properties [1], and which should also include an evaluation of the relative importance of temporal and spatial factors, such as when and where plastic debris and MPs are released to the environment. Mechanistic models are therefore needed to capture non-intuitive outcomes possibly arising from such interactions. In summary, putting the emphasis on MPs in the Perspective should not be interpreted as implying that the LRET of macroplastic is not important, as it clearly would be inconsistent with my support of the development and application of holistic approaches.

The concern raised regarding the limited attention given to the LRET of macroplastic may also be related to the discussion of the relative importance of land-based sources versus abandoned, lost, or otherwise discarded fishing gear (ALDFG). While Glüge et al. [2] provide an example that demonstrates the potential for both landbased and ALDFG sources of plastic debris to be subject to LRET (which I do not dispute) they seem to have misunderstood that discussion. Using a number of references [1], I attempted to draw attention to data which suggest that local sources of pollution are important contributors to the accumulation of plastic debris and MPs at various specific locations. Clearly, local sources cannot be ignored [1] and knowledge of proximal sources should be coupled with our understanding of distal plastic debris. A key message is that, given the ubiquitous use and release of plastic into the environment from both land-based and ocean-based sources, our understanding of 'remoteness' may require some redefinition [1]. Simply stated, buoyant plastic debris emitted to the environment can, under favourable conditions, indeed be subject to LRET, but there are also effective sinks that significantly limit the LRET of plastic debris and MPs [1]. The answer to whether or not MPs are subject to LRET is always 'it depends', since LRET is not just an intrinsic property of the plastic, it is system-dependent too. A better mechanistic understanding of the fate and transport of both plastic debris and MPs depends on characterizing and quantifying system-dependent properties [1]. Can models be used to characterize and quantify the mobility of plastic debris of varying shape, size, and density that might be released from a variety of both land-based and ocean-based sources? Can this information be used to help us understand the combination of processes and parameters that influence mobility? Given the emphasis of the Perspective [1] on the development and application of holistic mechanistic models, and which must include macroplastic and their degradation and fragmentation into MPs, it is not entirely clear how the concerns raised by Glüge et al. [2], which emphasizes the LRET of macroplastic, are sufficient to discredit the point of view expressed in the Perspective.

Next, Glüge et al. [2] express concern that the leaching of PACs from plastic debris does not receive sufficient attention and, therefore, the article fails to consider a significant component of the overall issue by not providing an in-depth assessment of leaching. Once again, it is useful to clarify the intention of the discussion presented. When considering the question 'does plastic represent a [substantial] source of exposure to PACs' to the environment [1], it seems appropriate to consider ALL potential emissions of PACs. Unlike the majority of studies that focus on the role of plastic debris and MPs as vectors of transport for PACs, I have attempted to place the issue into the context of releases of PACs occurring from products throughout their life-cycle. My calculations suggested that only about $3 \%$ of the total mass of PACs used in commerce up to 2015 is likely to still be present in plastic debris. Given that the remaining 97\% are continually being emitted to the environment and that plastic 
products are ubiquitous across the planet, it would therefore be prudent to evaluate the fate of, and exposure to, PACs being emitted from in-use and end-of-life lifecycle stages [1]. This would represent a more holistic approach as opposed to simply focusing on only one part of the product lifecycle (discarded macroplastic). Thus, in the interest of supporting the development and application of holistic mechanistic models, I proposed that the research community attempt to develop data that enable the characterization and quantification of all fate, transport and exposure pathways [1]. Glüge et al. [2] express concern that not enough attention is given to the leaching of PACs from MPs, such as into marine water or the digestive fluids of organisms that may have ingested them. However, the Perspective does not imply that these processes should be ignored. Simply that the relative importance of all process be placed into context by considering other relevant exposure pathways. Failing to consider all potential fate, transport and exposure pathways would be counterintuitive towards the adoption of a holistic approach.

Glüge et al. [2] imply that the Perspective does not acknowledge studies that have investigated the leaching of PACs and/or that the Perspective cites studies that are inappropriate in the context of PACs. In fact, the discussion of the leaching of PACs attempts to draw attention to what is already known by citing various standard methods and studies that have been published on the leaching of PACs from a variety of consumer products [1]. While the Perspective does not refer to the particular study identified by Glüge et al. [2] (i.e. Endo et al. [4]), it does include references to a number of other equally relevant studies, which yield similar insights regarding the time frame and rate limiting processes that influence leaching.

Another possible misunderstanding on the part of Glüge et al. [2] concerns my discussion about the role that particulate organic matter (POM) might play as an analogue for better understanding the LRET of MPs. This uses the example of POM as an analogy only illustrating the fate and transport of natural particles in aqueous systems. An analogy is an inference that if two or more things agree with one another in some respects, they will probably agree in others. While experience teaches that some analogies are successful at both explaining and predicting future scenarios, the use of analogy can also lead to error, especially if the analogies are inappropriate, as implied by Glüge et al. [2]. It cannot be claimed, however, that the use of analogy will lead us to an infallible theory, only that it can suggest a theory [5]. This is an important observation, especially when we move from well understood to poorly understood phenomena. Analogies, therefore, can be extremely helpful by providing information that can facilitate understanding of possible relationships [5]. Thus the question arises: when is an analogy appropriate, and when is it inappropriate? Glüge et al. [2] suggest that POM represents an inappropriate analogy because it is biodegradable. My perception, however, is that the value of the analogy remains an open question and I would not limit opportunities for scientific research to further explore the plausibility of the comparison. To limit scientific understanding by failing to evaluate the strengths and weaknesses that an analogy may represent, does not appear to be in the best interest of science.

Lastly, Glüge et al. [2] take issue with my unwarranted criticism of Andrade et al. [3]. In the Perspective [1] I simply proposed that understanding the fate, transport and exposure of PACs released to the environment requires an understanding of their use throughout the plastic life cycle, whereas Andrade et al. [3] appear to limit their assessment to the fate PACs associated with marine plastic debris. Ultimately, the added value generated by the Perspective [1] and the contribution of Andrade et al. [3] will be decided by the scientific community.

In preparing the Perspective [1], I fully anticipated and explicitly acknowledged that not everyone will agree with the point of view or perspective expressed. I am delighted, that it has already provoked some debate and constructive criticism. A comprehensive and quantitative picture of the importance of plastics in dispersing PACs over long distances and of the exposures of ecological and human receptors that it may cause cannot be achieved without the consideration of varying perspectives and the bundling of complementary expertise. The research community is attempting to address a complex environmental issue that requires active and constructive engagement from a variety of stakeholders and scientists from a wide range of backgrounds. Being open to alternative perspectives, in my opinion, should be encouraged. Ideally, a community that is open and respectful to varying points of view is better equipped to solve complex problems collectively. Collegial discourse thus represents an important component towards forming consensus, which is necessary to expedite effective and efficient solutions to the problem of LRET and to the issue of unwanted plastic debris and MPs in the environment.

\section{Abbreviations}

ALDFG: Abandoned, lost, or otherwise discarded fishing gear; LRET: Longrange environmental transport; MPs: Microplastic particles; PACs: plastic additive chemicals.

Acknowledgements

Not applicable . 


\section{Author's contributions}

The conception and design of the correspondence entirely written by TG. The author(s) read and approved the final manuscript.

\section{Funding}

No funding was received for this correspondence.

\section{Availability of data and materials}

Not applicable.

\section{Declarations}

\section{Competing interests}

TG declares that the preparation of this correspondence is not associated with any competing interests.

Received: 4 December 2021 Accepted: 7 January 2022

Published online: 27 January 2022

\section{References}

1. Gouin T. Addressing the importance of microplastic particles as vectors for long-range transport of chemical contaminants: perspective in relation to prioritizing research and regulatory actions. Microplastics and Nanoplastics. 2021;1(1):1-9.

2. Glüge J, Ashta NM, Herzke D, Lebreton L, Scheringer M. Letter to the editor regarding the perspective article "addressing the importance of microplastic particles as vectors for long-range transport of chemical contaminants: perspective in relation to prioritizing research and regulatory actions". Microplastics and Nanoplastics. in review.

3. Andrade H, Glüge J, Herzke D, Ashta NM, Nayagar SM, Scheringer M. Oceanic long-range transport of organic additives present in plastic products: an overview. Environmental sciences. Europe. 2021;33(1):1-4.

4. Endo S, Yuyama M, Takada H. Desorption kinetics of hydrophobic organic contaminants from marine plastic pellets. Mar Pollut Bull. 2013;74(1):125-31.

5. Hesse M. Models and analogies in science: University of Notre Dame Press; 1963. p. 190-1.

\section{Publisher's Note}

Springer Nature remains neutral with regard to jurisdictional claims in published maps and institutional affiliations.

\section{Submit your manuscript to a SpringerOpen ${ }^{\circ}$ journal and benefit from:}

- Convenient online submission

- Rigorous peer review

- Open access: articles freely available online

- High visibility within the field

- Retaining the copyright to your article 